\title{
Longitudinal Analysis of the Choriocapillaris Using Optical Coherence Tomography Angiography Reveals Subretinal Fluid as a Substantial Confounder in Patients with Acute Central Serous Chorioretinopathy
}

\author{
Michael Reich · Daniel Böhringer - Bertan Cakir · Felicitas Bucher • Moritz Daniel •
}

Stefan Lang • Wolf Lagrèze · Hansjürgen Agostini · Clemens Lange

Received: September 2, 2019 / Published online: October 25, 2019

(c) The Author(s) 2019

\section{ABSTRACT}

Introduction: To describe the influence of subretinal fluid (SRF) in analysis of the flow signal from the choriocapillaris (CC) via optical coherence tomography angiography (OCTA) in patients with acute central serous chorioretinopathy (CSC).

Methods: Observational, longitudinal, crosssectional case-control study. Twenty-three eyes of 22 patients with acute CSC were compared with 20 eyes of 20 healthy controls. OCTA images (AngioPlex ${ }^{\circledR}$, Zeiss) were recorded at baseline and follow-up examination and automatically quantified using an image-processing algorithm. Abnormal CC decorrelation signals (increased and decreased flow, IF and DF) were quantified using a custom image-processing algorithm. To analyze the spatial correlation between SRF and OCTA, CC signal heat-map images containing macular thickness information were used.

Enhanced Digital Features To view enhanced digital features for this article go to https://doi.org/10.6084/ m9.figshare.9948569.

M. Reich · D. Böhringer · B. Cakir · F. Bucher ·

M. Daniel · S. Lang · W. Lagrèze · H. Agostini ·

C. Lange $(\square)$

Eye Center, Medical Center, Faculty of Medicine,

University of Freiburg, Freiburg, Germany

e-mail: clemens.lange@uniklinik-freiburg.de
Results: Choriocapillaris flow alterations were significantly more pronounced at baseline than at follow-up examination in patients with acute CSC and resolving SRF (IF: $p<0.002 ;$ DF: $p<0.003)$. The extent of SRF correlated significantly with the abnormal CC decorrelation signals when comparing baseline with followup examinations (IF: rho $=0.56, p<0.005$; DF: rho $=0.64, p<0.001)$. In particular, the reduced OCTA flow signal in the area occupied by SRF at baseline disappeared at follow-up examination $(p<0.02)$. In comparison with controls, CSC patients with complete resorption of SRF continued to show a reduced OCTA flow signal in the CC $(p<0.008)$.

Conclusions: The temporospatial association between SRF and reduced OCTA signal from the CC demonstrates that fluid might be a significant confounder for OCTA analysis in patients with CSC. CSC patients with complete absorption of the SRF at follow-up, however, show persistently reduced CC flow signals.

Keywords: Artifacts; Central serous chorioretinopathy; Choriocapillaris; OCT angiography; Subretinal fluid

\section{Abbreviations}

$\Delta \mathrm{RT}$

Difference in average retinal thickness between baseline and follow-up examination

AMD Age-related macular degeneration 


\begin{tabular}{|c|c|}
\hline BCVA & Best-correlated visual acuity \\
\hline BL & Baseline \\
\hline $\mathrm{CC}$ & Choriocapillaris \\
\hline $\mathrm{CSC}$ & $\begin{array}{l}\text { Central serous chorioretino- } \\
\text { pathy }\end{array}$ \\
\hline DF & Decreased flow signal \\
\hline FU & Follow-up \\
\hline IF & Increased flow signal \\
\hline ICGA & Indocyanine green angiography \\
\hline OCTA & $\begin{array}{l}\text { Optical coherence tomography } \\
\text { angiography }\end{array}$ \\
\hline RPE & Retinal pigment epithelium \\
\hline SD-OCT & $\begin{array}{l}\text { Spectral domain optical } \\
\text { coherence tomography }\end{array}$ \\
\hline Spearman's $R$ & $\begin{array}{l}\text { Spearman's rank correlation } \\
\text { coefficient }\end{array}$ \\
\hline SRF & Subretinal fluid \\
\hline $\mathrm{w} / \mathrm{o}$ & Without \\
\hline
\end{tabular}

\section{Key Summary Points}

Why carry out this study?

There is a lack of follow-up data in the literature regarding the choriocapillaris analyses via OCTA in acute central serous chorioretinopathy.

It was this study's aim to describe the influence of subretinal fluid in analysing the flow signal from the choriocapillaris via OCTA, and to describe the flow alteration after subretinal fluid absorption in acute central serous chorioretinopathy.

\section{What was learned from the study?}

The extent, respectively decrease of subretinal fluid correlates with the abnormal choriocapillaris decorrelation signal.

Subretinal fluid is a distinct confounder when analysing the choriocapillaris via OCTA.

Central serous chorioretinopathy patients exhibiting complete absorption of subretinal fluid at follow-up show persistent choriocapillary changes.

\section{INTRODUCTION}

Central serous chorioretinopathy (CSC) is a common vision-threatening maculopathy with an incidence of 1:10,000 [1]. It is characterized by localized subretinal fluid (SRF) which selfresolves in most of cases within 6 months [2]. The pathogenesis of CSC is still poorly understood. Recent studies suggest that choriocapillaris (CC) endothelial cell dysfunction is the primary cause of the disease, leading to secondary retinal pigment epithelium (RPE) dysfunction [3, 4]. While conventional imaging techniques such as fluorescein and indocyanine green angiography can only partially depict the $\mathrm{CC}$, optical coherence tomography angiography (OCTA) enables the assessment and quantification of CC blood flow [5]. OCTA is a noninvasive technology that uses laser light reflectance of the surface of moving red blood cells to depict blood flow in the retinal and choroidal microvasculature [6,7]. This technology has enhanced our understanding of microvascular alterations in various macular diseases including diabetic retinopathy, retinal vein occlusion, and age-related macular degeneration [5]. However, OCTA is a light sourcedependent device with considerable limitations. Shadowing artifacts caused by media opacities $[8,9]$ or superficial blood vessels blocking the detection of deeper vessels in the retina can compromise the analysis [10]. Similarly, projection artifacts caused by traversing light reflected by deeper layers (e.g., RPE) can mimic a vascular pattern in those retinal layers and thus confound the analysis [11].

Since its introduction, several groups have used OCTA to investigate the CC in patients with CSC [12-19]. However, none of these studies provides follow-up data in acute CSC assessing the OCTA signal at the level of the CC when SRF is absorbed. Therefore, the aim of this study was to describe the influence of SRF in the analysis of the flow signal from the CC via OCTA, and to describe the flow alteration after SRF absorption in acute CSC. 


\section{METHODS}

\section{Study Design and Population}

This longitudinal, cross-sectional, single-center observational case-control study was conducted in 22 consecutive patients with acute CSC (23 eyes). Only patients with classic acute CSC defined by a dome-shaped accumulation of central SRF, no pronounced RPE changes or RPE detachments, and symptoms lasting less than 4 months were included in the study [20]. Data from the baseline examinations of 17 patients were previously published by Cakir et al. [12]. Twenty age-matched and healthy participants with best-corrected visual acuity (BCVA) of $20 / 20$ or better and no chorioretinal pathology served as controls. All patients consulted our clinic between 2016 and 2018. Follow-up examinations were performed individually according to the disease course. No study participant had any concomitant ocular disease with CSC, or prior ophthalmologic surgery or laser treatment. In particular, there was no evidence of acquired or hereditary macular disease. Patient characteristics including gender, date of birth, pre-existing conditions, initial diagnosis, previous treatment, and duration of symptoms were recorded. This study was performed in accordance with the ethical standards of the ethics committee of the University of Freiburg (application no. 561/16) and with the 1964 Helsinki Declaration and its later amendments or comparable ethical standards. Written informed consent was obtained from all patients and controls for the publication of this study and any accompanying images.

\section{Ophthalmological Examination}

All patients underwent a thorough ophthalmological examination including measurement of BCVA, assessment of intraocular pressure, biomicroscopy of the anterior segment, and ophthalmoscopy after dilation with tropicamide $1 \%$ eye drops. Additionally, all patients underwent spectral-domain (SD)-OCT and fundus autofluorescence imaging (Spectralis HRA; Heidelberg Engineering, Heidelberg, Germany). OCTA examinations were performed using the commercially available Zeiss Cirrus 5000 AngioPlex ${ }^{\circledR}$. It uses full-spectrum spectral domain (SD-OCT) with a light-source wavelength of $840 \mathrm{~nm}$, an A-scan rate of 68,000 A-scans per second, and an A-scan depth of $2.0 \mathrm{~nm}$ in tissue (1024 pixels). The built-in software was used to eliminate artifacts created from superficial vascular layers. Each patient underwent a $6 \times 6 \mathrm{~mm}^{2}$ volume scan. For OCTA visualization of the CC layer, a $30 \mu \mathrm{m}$ slab between 29 and $59 \mu \mathrm{m}$ below the inner RPE was manually selected for each patient.

Abnormal CC decorrelation signals were quantified using an image-processing algorithm programmed in R (http://www.r-project.org) as described previously [12, 13]. Briefly, images were processed via a Gaussian blur and morphological hdome-operator, and the average grayscale value was determined. The threshold for the color-coding was calculated for each OCTA image using the averaged grayscale value of all pixels (brightness of pixel) plus (increased flow, red pixels) or minus (decreased flow, green pixels) a constant and predefined threshold value. Before quantifying the pixel counts, shadowing artifacts of the inner retinal vessels were manually removed from each image. The pixel counts were used for statistical analyses. The average retinal thickness of the $6 \times 6 \mathrm{~mm}^{2}$ volume scan was measured via the heat-map image containing macular thickness information obtained from the Zeiss Cirrus 5000 AngioPlex $^{\circledR}$.

To explore a possible longitudinal correlation between SRF alterations and the CC flow signal over time, the difference in average retinal thickness $(\Delta \mathrm{RT})$ between baseline $(\mathrm{BL})$ and follow-up (FU) examination was correlated with the difference in increased and decreased OCTA flow signal.

To analyze the potential vascular change in the CC in the area previously occupied by SRF and with no SRF at the time of follow-up 
examination, the color-coded CC images were aligned with the corresponding heat-map images. The area revealing macular thickness exceeding $450 \mu \mathrm{m}$ was defined as the region of interest and automatically outlined by the software. Red and green pixel counts within and outside the area were recorded and used for comparison with the control group. For statistical analyses, the percentage of the area with decreased versus increased flow signal was used.

\section{Statistical Methods}

Statistical analysis was performed using GraphPad Prism 6 (GraphPad Software, Inc., La Jolla, CA, USA). The Mann-Whitney test was used to compare paired samples, and the Wilcoxon test was performed to compare unpaired samples. Bonferroni correction due to multiple comparisons was included. Spearman's rank correlation coefficient (Spearman's $R$ ) was used to determine the relationship between SRF and OCTA flow signal. Data were considered significant at $p<0.05$.

\section{RESULTS}

\section{Patient Characteristics}

A total of 23 eyes from 22 patients with acute CSC (male/female 19/3) and 20 eyes from 20 controls (male/female 10/10) were included in this study. The average age of the CSC population was 51.2 years (range 37-80), compared with 49.9 years (range 22-79) in the control group $(p=0.93)$. The time from the first symptom to the first examination in our hospital ranged from 0 to 86 days (average $20.2 \pm 29.6$ days). After the initial diagnosis, 10 patients had been treated with eplerenone, whereas 12 patients had not been treated with any systemic or topical medication. Follow-up examination was performed $134 \pm 112$ days after baseline examination. In 13 eyes (12 patients), SRF was completely absorbed at the follow-up examination. Five of these patients had been treated with eplerenone, while seven remained untreated.

\section{Longitudinal Correlation Between Subretinal Fluid Alterations and the Signal of Flow of the CC Over Time}

In the 19 eyes in which average retinal thickness was decreased at follow-up (BL: $334.5 \pm$ $29.3 \mu \mathrm{m}$, FU: $298.9 \pm 20.8 \mu \mathrm{m}, p<0.0001$ ), we observed a significant reduction in CC alterations at follow-up compared with baseline examination (Fig. 1a, b). The area of increased CC flow signal was $3.5 \%( \pm 1.4 \%)$ at baseline and $2.04 \%( \pm 1.1 \%)$ at follow-up examination $(p<0.002)$. Similarly, the area of reduced flow signal decreased from $2.5 \%( \pm 1.4 \%)$ at baseline to $1.10 \%( \pm 0.6 \%)$ at follow-up examination $(p<0.003)$. However, in the four eyes in which retinal thickness increased over time (retinal thickness at BL: $308.6 \pm 11.7 \mu \mathrm{m}, \quad$ FU: $338.2 \pm 26.8 \mu \mathrm{m}, p=0.08$ ), we found no obvious difference in the area of CC flow changes at follow-up relative to the baseline examination. The areas of both increased CC flow signal $(2.5 \% \pm 1.7 \%)$ and decreased CC flow signal $(2.3 \% \pm 2.2 \%)$ were similar between the baseline and follow-up examinations $(3.8 \% \pm 2.4 \%$ and $3.5 \% \pm 3.3, p=0.56$ and $p=0.25$ ). Interestingly, the area of diminished OCTA signal shifted below the center at follow-up examination, which was associated with a downward shift of the SRF (Fig. 1c, d).

To explore the correlation between SRF and the OCTA flow signal of the CC, we correlated the difference in average retinal thickness on the $6 \times 6 \mathrm{~mm}^{2}$ volume scan between baseline and follow-up examination with the difference in detected OCTA flow signal from the CC. Using the Spearman rank correlation coefficient test, we found that the extent of SRF correlated significantly with the CC flow alterations (decreased flow: rho $=0.64, p<0.001$, increased flow: rho $=0.56, p<0.005$, Fig. 1e, f).

\section{Change in the OCTA Signal \\ from the Choriocapillaris After Complete Absorption of SRF}

To analyze the OCTA signals in the CC following SRF absorption, 13 eyes presenting complete absorption of SRF at follow-up were evaluated. 

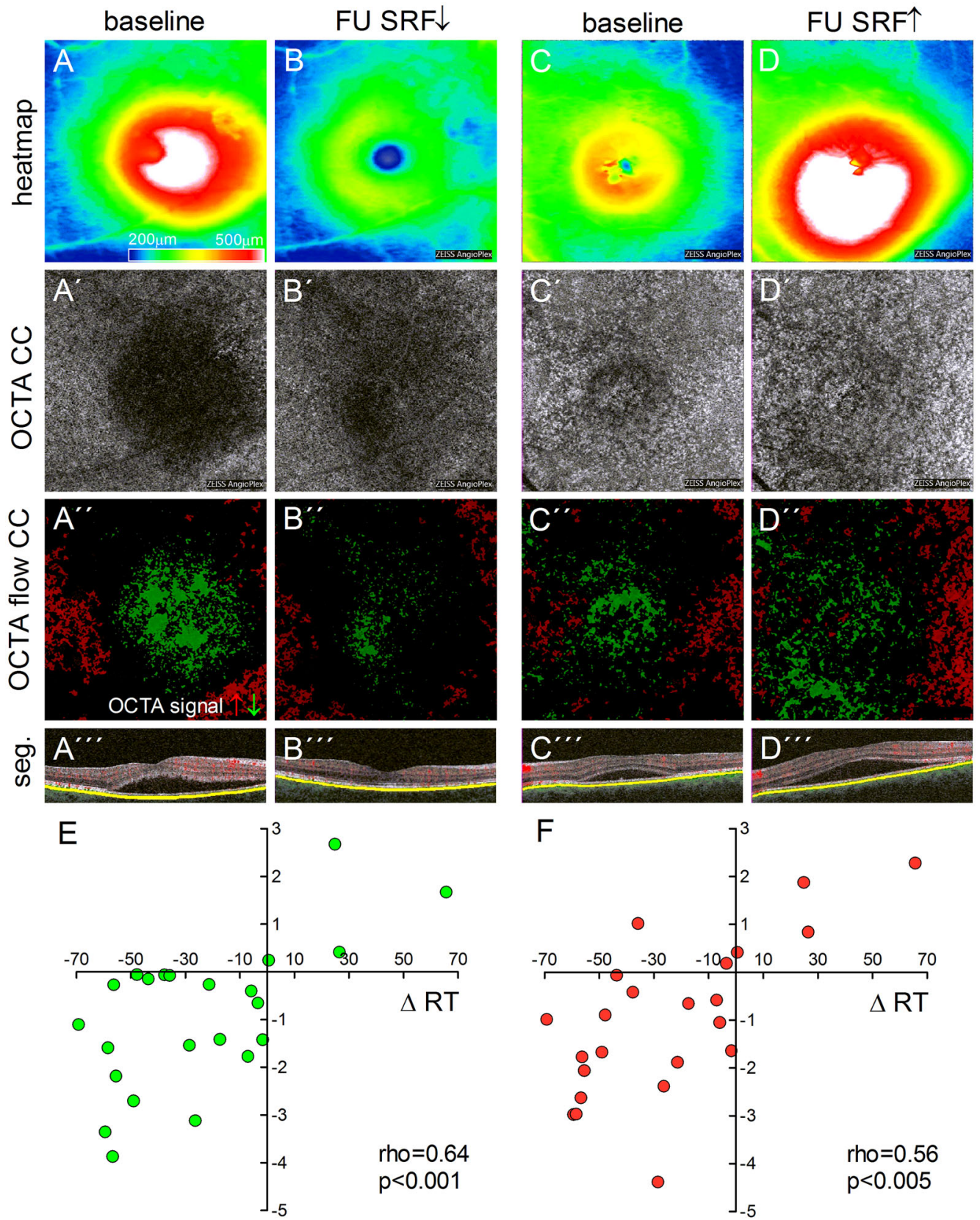

\section{$\Delta$ of decreased OCTA signal}

Fig. 1 A decrease in subretinal fluid is associated with a reduction in OCTA choriocapillaris alteration. Representative retinal thickness heat maps (top row) and OCTA images of the choriocapillaris (CC) without and with flow pseudo-coloration (middle rows) and OCTA segmentation (bottom row) in patients with acute central serous chorioretinopathy (CSC) demonstrating decreasing (a, b) or increasing (c, d) subretinal fluid (SRF) at follow-up (FU) examination compared with baseline. Decreased flow signal from the CC is illustrated with green pixels,

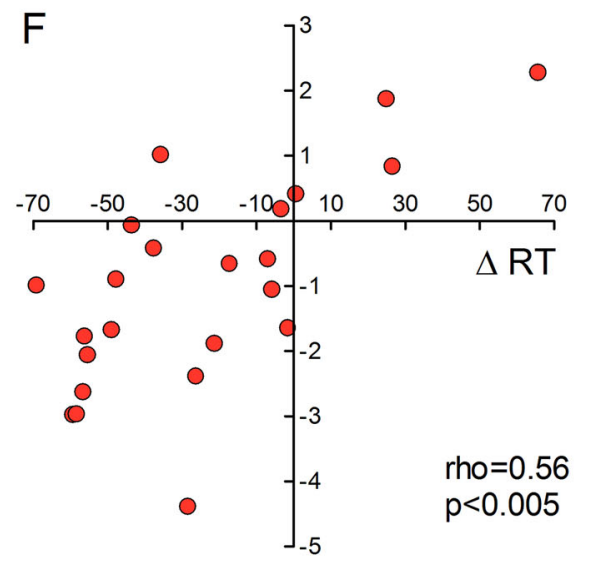

\section{$\Delta$ of increased OCTA signal}

increased flow signal with red pixels. a, b The SRF spontaneously absorbed at FU examination, which is associated with a reduction in decreased CC OCTA flow signal. c, d SRF at FU examination is markedly increased, which is associated with a decrease in CC OCTA flow signal. e, $\mathbf{f}$ Retinal thickness differences between baseline and follow-up $(\Delta \mathrm{RT}$ in $\mu \mathrm{m})$ correlate with increasing or decreasing flow signal (presented in \% of total area). Each dot represents one eye. rho $=$ Spearman's $R$ 

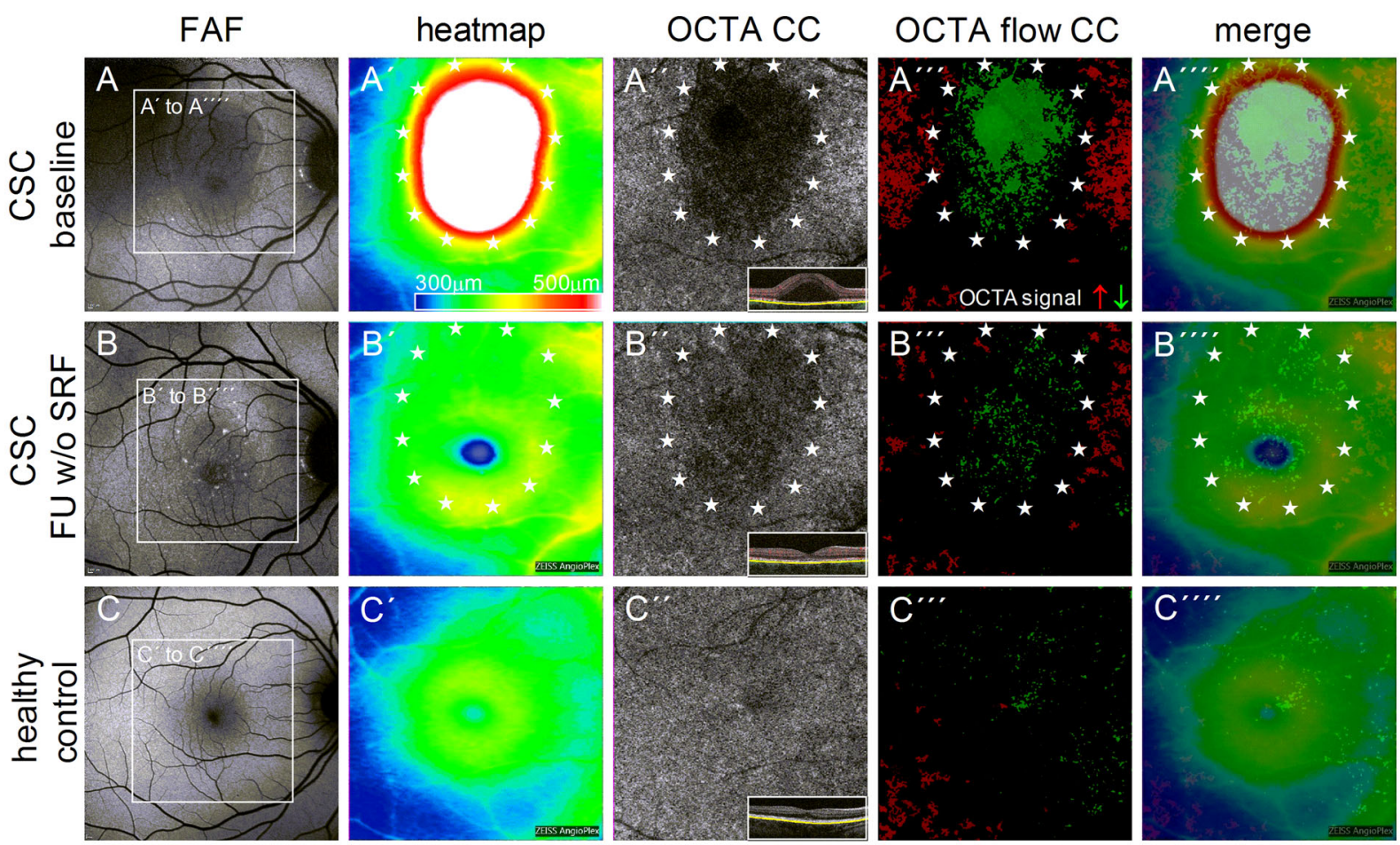

D Analysis of the entire image $6 \times 6 \mathrm{~mm}^{2}$

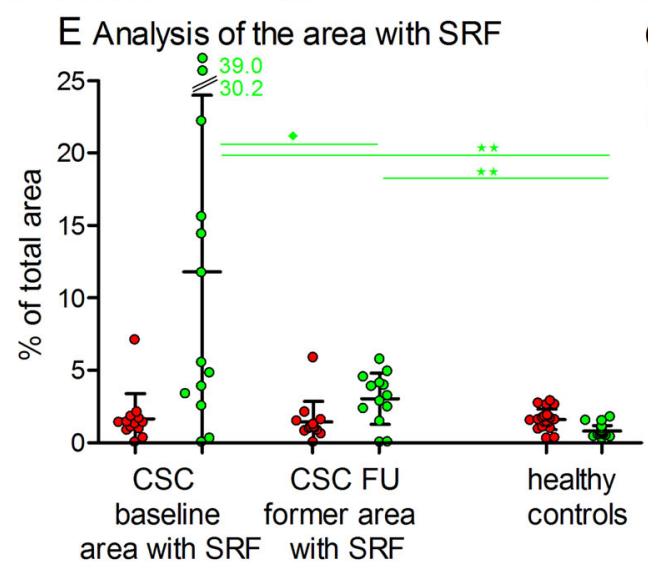

OCTA signal

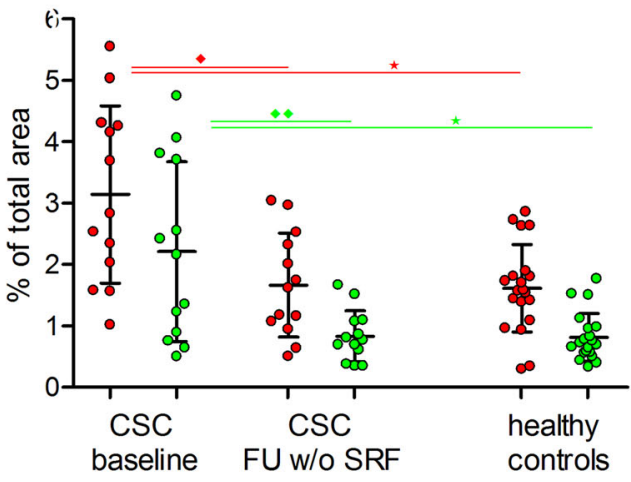

increased

decreased

Fig. 2 Patients with central serous chorioretinopathy exhibiting complete absorption of subretinal fluid at follow-up show persistent choriocapillaris changes. Representative images of a patient with active central serous chorioretinopathy (CSC) at baseline $\left(\mathbf{a}-\mathbf{a}^{\prime \prime \prime \prime}\right)$ and complete absorption of subretinal fluid (SRF) at follow-up examination (FU, b- $\left.\mathbf{b}^{\prime \prime \prime \prime}\right)$ compared with healthy control $\left(\mathbf{c}-\mathbf{c}^{\prime \prime \prime \prime}\right)$. Representative fundus autofluorescence (FAF, first column), retinal thickness heat maps (second column), OCTA images of the choriocapillaris (CC) without and with flow pseudo-coloration (middle columns), and a merged image (last column) are shown. Decreased flow

signal from the CC is illustrated with green pixels, increased flow signal with red pixels. Asterisks surround the area of SRF at baseline examination defined as $>450 \mu \mathrm{m}$ macular thickness $\left(\mathbf{a}^{\prime}\right) . N=13$ patients. d, e Quantitative analysis of OCTA flow changes in the entire $6 \times 6 \mathrm{~mm}$ image (d) and the area with SRF at baseline (e). Each dot represents one eye. Wilcoxon test including Bonferroni correction due to multiple comparisons was performed to compare unpaired samples $p<$ $0.05,{ }^{*} p<0.01$. Mann-Whitney test including Bonferroni correction due to multiple comparisons was used to compare paired samples ${ }^{*} p<0.05,{ }^{* *} p<0.01$ 
Table 1 Change in the OCTA flow signal from the choriocapillaris in patients with acute central serous chorioretinopathy (CSC) after complete absorption of subretinal fluid (SRF)

\begin{tabular}{llc}
\hline & $\begin{array}{l}\text { Increased flow } \\
\text { signal }\end{array}$ & $\begin{array}{l}\text { Decreased } \\
\text { flow signal }\end{array}$ \\
\hline $\begin{array}{l}\text { Healthy controls } \\
\text { CSC baseline }\end{array}$ & $1.62 \pm 0.71$ & $0.81 \pm 0.39$ \\
\% of total area & $3.14 \pm 1.44$ & $2.21 \pm 1.46$ \\
\% of the area with & $1.65 \pm 1.73$ & $11.80 \pm 12.20$ \\
SRF & & \\
\% of the area w/o & $5.48 \pm 3.07$ & $1.88 \pm 1.26$ \\
SRF & & \\
$\begin{array}{l}\text { CSC follow-up w/o SRF } \\
\text { \% of total area }\end{array}$ & $1.66 \pm 0.85$ & $0.83 \pm 0.41$ \\
\% of the former area & $1.43 \pm 1.42$ & $3.04 \pm 1.77$ \\
with SRF & & \\
\% of the former area & $2.73 \pm 1.55$ & $0.91 \pm 0.55$ \\
w/o SRF & & \\
\hline
\end{tabular}

Looking at the entire $6 \times 6 \mathrm{~mm}^{2}$ OCTA image, the OCTA signal alterations were greater at the baseline examination compared with the follow-up examination (increased flow: $p<0.028$, decreased flow: $p<0.009$ ) and with healthy controls (increased flow: $p<0.039$, decreased flow: $p<0.039$; Fig. 2d, Table 1). No statistically significant difference was found in the decorrelation signals between CSC patients without SRF and healthy controls when the entire image was analyzed. However, when looking at the area where the SRF was initially located, the decreased OCTA flow signal persisted and was significantly more pronounced than in healthy controls despite the absence of SRF $(p<0.008$; Fig. 2e, Table 1). These data point toward localized CC changes that are spatially correlated with the area of SRF in acute CSC and persist in inactive CSC, independent of SRF. The detailed statistical results for the analysis of changes in the OCTA flow signal from the CC in patients with acute CSC after complete SRF absorption are listed in Table 2.

\section{DISCUSSION}

Optical coherence tomography angiography (OCTA) is a promising noninvasive technique for imaging the microvasculature of the retina and choroid. However, artifacts occurring in OCTA, such as shadowing artifacts caused by intra- or subretinal fluid (SRF), may lead to misdiagnosis and unnecessary treatment $[9,11,21]$. In this longitudinal OCTA study, we demonstrate a strong temporospatial association between SRF and reduced OCTA signals from the choriocapillaris (CC) in patients with acute CSC, indicating that SRF might be a significant confounder for the OCTA analysis of the CC.

Several studies have shown that the OCTA signal of the CC is reduced in patients with CSC, interpreted as reduced blood flow of the CC $[14,16-18]$. Although some authors have suspected that SRF contributes to the reduced OCTA flow signal in the CC in the sense of a shadow artifact [12, 22], this could not be demonstrated due to lack of follow-up data. Our longitudinal study shows that CSC patients with complete absorption of SRF at follow-up demonstrate a significant reduction in OCTA flow alterations in the CC, indicating that SRF prevents the detection of OCTA signals from the CC. In line with this finding, patients with persistent and vertically displaced SRF at the follow-up examination exhibited a reduced OCTA signal from the CC in the affected area. These findings support the prevailing hypothesis that SRF is a disruptive factor in the analysisof CC via OCTA and that the quantitativeanalysis of CC flow changes in the presence of retinal fluid must be considered with caution $[5,12]$. This applies not only to CSC, but also to other retinal vascular diseases associated with subretinal or intraretinal fluid, such as neovascular age-related macular degeneration (AMD). For example, in geographic atrophy in AMD, it has been assumed and histologically demonstrated that RPE atrophy is associated with CC atrophy [23, 24]. CC OCTA studies, correspondingly, have shown decreased CC flow in areas of RPE atrophy $[25,26]$. Similarly, CC OCTA studies in patients with 


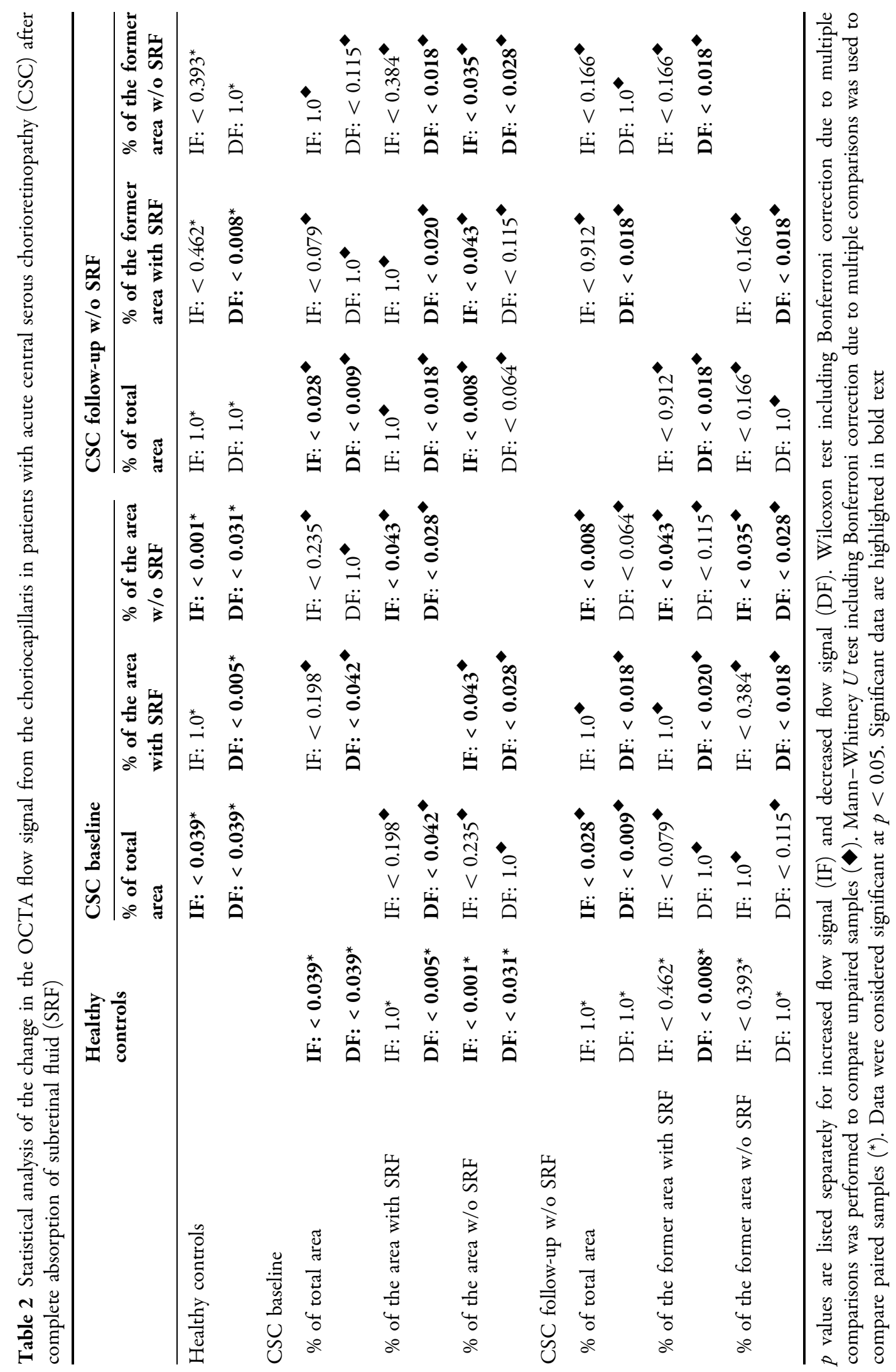


intermediate and subclinical exudative AMD have postulated the existence of CC signal voids $[27,28]$. Considering our results, especially in the description and quantification of CC signal voids in patients with neovascular AMD, intraor subretinal fluid above this area must be excluded with confidence [29]. Further studies investigating this hypothesis are needed and must exclude any intraretinal or subretinal fluids which, as shown in this study, can significantly affect OCT CC and potentially even mimic CC signal voids.

By following up patients with acute CSC until the absorption of SRF, our study bypasses the above-mentioned shadowing effect of SRF on the OCTA flow signal, and a more reliable conclusion about possible vascular changes in the CC can be drawn. Interestingly, we found that the reduced OCTA signal in the CC persisted after SRF absorption, indicating a reduced blood flow in the CC in CSC patients that is spatially associated with the area of SRF. This hypothesis is in line with previous OCTA CC studies describing areas of low flow signal in CSC patients [12, 15-17] which may indicate a thinned CC pushed upward by pachyvessels or low blood flow at the CC level [14, 22]. Studies applying indocyanine green angiography (ICGA) support this assumption [4, 30, 31] and show delayed choroidal filling on ICGA at the site of the hot spot in $82 \%$ of CSC patients [32] and a $45 \%$ reduction in choroidal blood flow in CSC eyes compared with unaffected fellow eyes [33]. A comparison of OCTA and ICGA demonstrated that areas with coarse low-reflective OCTA signal corresponded with the area of hypo-fluorescence on early-phase ICGA [22]. These studies support the findings of our study and indicate that CSC is associated with reduced choroidal blood blow.

We acknowledge that this study has some limitations, including its retrospective design and small number of patients. Although agematched controls were used, we did not match patients regarding refraction or length of bulbus, nor did we collect blood pressure or antihypertensive medication data, both of which might be confounders in our study. Although shadowing artifacts by SRF were excluded, artifacts are potentially caused by RPE and photoreceptor outer segment irregularities as well as RPE detachment [34, 35]. Since pronounced RPE changes after one episode of acute CSC are mild and are not located exclusively in the area previously occupied by SRF, a shadowing artifact on the OCTA flow signal from the CC of these RPE changes seems unlikely, but cannot be entirely excluded. Another limitation of our study is the use of the averaged pixel intensity of each image to determine increased and decreased flow changes within the same image. Therefore, local, large deviations in the flow signal influence the average signal, which may have caused some over- and underestimation of flow changes. Prospective longitudinal studies with optimized image acquisition techniques less prone to artifacts are therefore necessary to further validate our findings.

\section{CONCLUSIONS}

In summary, this longitudinal OCTA study reports follow-up data from patients with acute CSC during their initial episode of CSC until SRF was completely removed. Although we demonstrate that SRF is a distinct confounder when analyzing the CC via OCTA, we also reveal that the OCTA flow signal from the CC within the area previously occupied by SRF continues to be reduced after SRF has completely resolved compared with that in healthy controls, indicating reduced blood flow in patients with acute CSC.

\section{ACKNOWLEDGEMENTS}

We thank the participants of the study.

Funding. No funding or sponsorship was received for this study or publication of this article. The Rapid Service Fee was funded by the authors.

Authorship. All named authors meet the International Committee of Medical Journal Editors (ICMJE) criteria for authorship for this article, take responsibility for the integrity of 
the work as a whole, and have given their approval for this version to be published.

Authorship Contributions. Michael Reich designed the study, made substantial contributions to the acquisition, analysis and interpretation of the data and preparation of the manuscript, and is the lead author of the manuscript. Daniel Böhringer made substantial contributions to acquisition of the data, and was involved in drafting the manuscript. Bertan Cakir made substantial contributions to acquisition of the data and was involved in drafting the manuscript. Moritz Daniel made substantial contributions to interpretation of the data and was involved in drafting the manuscript. Felicitas Bucher made substantial contributions to interpretation of the data and was involved in drafting the manuscript. Stefan Lang made substantial contributions to interpretation of the data and was involved in drafting the manuscript. Wolf Lagrèze made substantial contributions to interpretation of the data and was involved in drafting the manuscript. Hansjürgen Agostini made substantial contributions to interpretation of the data and was involved in critically revising the manuscript for important intellectual content. Clemens Lange made substantial contributions to the analysis and interpretation of the data and preparation of the manuscript, and was involved in critically revising the manuscript for important intellectual content. In addition, all authors have given final approval of the version to be published. Each author participated sufficiently in the work to take public responsibility for appropriate portions of the content and agree to be accountable for all aspects of the work in ensuring that questions related to the accuracy or integrity of any part of the work are appropriately investigated and resolved. All authors read and approved the final manuscript.

Disclosures. Daniel Böhringer: Boehringer Ingelheim, Novartis, Orphan Europe. Hansjürgen Agostini: Allergan, Novartis, Roche, Zeiss, Bayer. Michael Reich, Bertan Cakir, Moritz Daniel, Felicitas Bucher, Wolf Lagrèze, and
Clemens Lange declare that they have no competing interests.

Compliance with Ethics Guidelines. This study was performed in accordance with the ethical standards of the ethics committee of the University of Freiburg (application no. 561/16) and with the 1964 Helsinki Declaration and its later amendments or comparable ethical standards. Written informed consent was obtained from all patients and controls for the publication of this study and any accompanying images. The study does not contain any personal information that could lead to the identification of the patients.

Data Availability. The data sets generated and/or analyzed during the current study are available from the corresponding author on reasonable request.

Open Access. This article is distributed under the terms of the Creative Commons Attribution-NonCommercial 4.0 International License (http://creativecommons.org/licenses/ by-nc/4.0/), which permits any noncommercial use, distribution, and reproduction in any medium, provided you give appropriate credit to the original author(s) and the source, provide a link to the Creative Commons license, and indicate if changes were made.

\section{REFERENCES}

1. Wang M, Munch IC, Hasler PW, Prunte C, Larsen M. Central serous chorioretinopathy. Acta Ophthalmol. 2008;86(2):126-45.

2. Daruich A, Matet A, Marchionno L, De Azevedo JD, Ambresin A, Mantel I, Behar-Cohen F (2017) Acute central serous chorioretinopathy: factors influencing episode duration. Retina 37(10):1905-1915. https://doi.org/10.1097/IAE.0000000000001443

3. Yoshioka H, Katsume Y. Experimental central serous chorioretinopathy. III: ultrastructural findings. Jpn J Ophthalmol. 1982;26(4):397-409.

4. Piccolino FC, Borgia L. Central serous chorioretinopathy and indocyanine green angiography. Retina. 1994;14(3):231-42. 
5. Kashani AH, Chen CL, Gahm JK, et al. Optical coherence tomography angiography: a comprehensive review of current methods and clinical applications. Prog Retin Eye Res. 2017;60:66-100.

6. Fingler J, Readhead C, Schwartz DM, Fraser SE. Phase-contrast OCT imaging of transverse flows in the mouse retina and choroid. Invest Ophthalmol Vis Sci. 2008;49(11):5055-9.

7. Koustenis A Jr, Harris A, Gross J, et al. Optical coherence tomography angiography: an overview of the technology and an assessment of applications for clinical research. $\mathrm{Br} \mathrm{J}$ Ophthalmol. 2017;101(1):16-20.

8. Spaide RF, Klancnik JM Jr, Cooney MJ. Retinal vascular layers imaged by fluorescein angiography and optical coherence tomography angiography. JAMA Ophthalmol. 2015;133(1):45-50.

9. Enders C, Lang GE, Dreyhaupt J, et al. Quantity and quality of image artifacts in optical coherence tomography angiography. PLoS One. 2019;14(1):e0210505.

10. Jia Y, Tan O, Tokayer J, et al. Split-spectrum amplitude-decorrelation angiography with optical coherence tomography. Opt Express. 2012;20(4):4710-25.

11. Spaide RF, Fujimoto JG, Waheed NK. Image artifacts in optical coherence tomography angiography. Retina. 2015;35(11):2163-80.

12. Cakir B, Reich M, Lang S, et al. OCT angiography of the choriocapillaris in central serous chorioretinopathy: a quantitative subgroup analysis. Ophthalmol Ther. 2019;8(1):75-86.

13. Cakir B, Reich M, Lang SJ, et al. Possibilities and limitations of OCT-angiography in patients with central serous chorioretinopathy. Klin Monbl Augenheilkd. 2017;234(9):1161-8.

14. Shinojima A, Kawamura A, Mori R, Fujita K, Yuzawa M. Findings of optical coherence tomographic angiography at the choriocapillaris level in central serous chorioretinopathy. Ophthalmologica. 2016;236(2):108-13.

15. Feucht N, Maier M, Lohmann CP, Reznicek L. OCT angiography findings in acute central serous chorioretinopathy. Ophthalmic Surg Lasers Imaging Retina. 2016;47(4):322-7.

16. Costanzo E, Cohen SY, Miere A, et al. Optical coherence tomography angiography in central serous chorioretinopathy. J Ophthalmol. 2015;2015:134783.
17. Teussink MM, Breukink MB, van Grinsven MJ, et al. OCT angiography compared to fluorescein and indocyanine green angiography in chronic central serous chorioretinopathy. Invest Ophthalmol Vis Sci. 2015;56(9):5229-37.

18. Chan SY, Wang Q, Wei WB, Jonas JB. Optical coherence tomographic angiography in central serous chorioretinopathy. Retina. 2016;36(11):2051-8.

19. Rochepeau C, Kodjikian L, Garcia MA, Mathis T. Optical coherence tomography angiography quantitative assessment of choriocapillaris blood flow in central serous chorioretinopathy. Am J Ophthalmol. 2018;194:26-34.

20. Daruich A, Matet A, Dirani A, et al. Central serous chorioretinopathy: recent findings and new physiopathology hypothesis. Prog Retin Eye Res. 2015;48:82-118.

21. Ghasemi Falavarjani K, Al-Sheikh M, Akil H, Sadda SR. Image artefacts in swept-source optical coherence tomography angiography. Br J Ophthalmol. 2017;101(5):564-8.

22. $\mathrm{Hu}$ J, Qu J, Piao Z, et al. Optical coherence tomography angiography compared with indocyanine green angiography in central serous chorioretinopathy. Sci Rep. 2019;9(1):6149.

23. McLeod DS, Grebe R, Bhutto I, et al. Relationship between RPE and choriocapillaris in age-related macular degeneration. Invest Ophthalmol Vis Sci. 2009;50:4982-91.

24. Saint-Geniez M, Kurihara T, Sekiyama E, et al. An essential role for RPE-derived soluble VEGF in the maintenance of the choriocapillaris. Proc Natl Acad Sci USA. 2009;106:18751-6.

25. Kvanta A, de Salles MC, Amrén U, Bartuma H. Optical coherence tomography angiography of the foveal microvasculature in geographic atrophy. Retina. 2017;37:936-42.

26. Pellegrini M, Acquistapace A, Oldani M, et al. Dark atrophy: an optical coherence tomography angiography study. Ophthalmology. 2016;123:1879-86.

27. Vujosevic S, Toma C, Villani E, et al. Quantitative choriocapillaris evaluation in intermediate age-related macular degeneration by swept-source optical coherence tomography angiography. Acta Ophthalmol. 2019;97(6):e919-26.

28. Treister AD, Nesper PL, Fayed AE, et al. Prevalence of subclinical CNV and choriocapillaris nonperfusion in fellow eyes of unilateral exudative AMD on OCT angiography. Trans Vis Sci Tech. 2018;7(5):19. 
29. Pilotto E, Frizziero L, Daniele AR, et al. Early OCT angiography changes of type $1 \mathrm{CNV}$ in exudative AMD treated with anti-VEGF. Br J Ophthalmol. 2019;103(1):67-71.

30. Hayashi K, Hasegawa Y, Tokoro T. Indocyanine green angiography of central serous chorioretinopathy. Int Ophthalmol. 1986;9(1):37-41.

31. Gass JD. Pathogenesis of disciform detachment of the neuroepithelium. Am J Ophthalmol. 1967;63(3):1-139.

32. Scheider A, Hintschich C, Dimitriou S. Central serous chorioretinopathy. Studies of the site of the lesion with indocyanine green. Ophthalmologe. 1994;91(6):745-51.
33. Kitaya N, Nagaoka T, Hikichi T, et al. Features of abnormal choroidal circulation in central serous chorioretinopathy. $\mathrm{Br} \quad \mathrm{J}$ Ophthalmol. 2003;87(6):709-12.

34. Spaide RF. Choriocapillaris flow features follow a power law distribution: implications for characterization and mechanisms of disease progression. Am J Ophthalmol. 2016;170:58-67.

35. Nesper PL, Soetikno BT, Fawzi AA. Choriocapillaris nonperfusion is associated with poor visual acuity in eyes with reticular pseudodrusen. Am J Ophthalmol. 2017;174:42-55. 